\title{
Challenges in the development of novel anticryptococcal agents
}

\author{
"...although there are many challenges inherent in the quest to \\ improve current methods in cryptococcal therapy, future prospects for \\ solving this problem seem more promising..."
}

First draft submitted: 19 May 2016; Accepted for publication: 6 June 2016; Published online: 3 August 2016

Keywords: antifungal • cryptococcus • HIV-AIDS-related infection

Based on a number of recently published estimates, human infections caused by Cryptococcus spp. represent the most prevalent life-threatening fungal infections facing our world [1,2]. Despite this prominence among invasive fungal infections, the current gold standard therapy for cryptococcal meningitis, the most common manifestation of cryptococcosis, is based on two drugs, amphotericin B and 5-flucytosine, that were developed in the late 1950s and early 1960s. Unfortunately, there are a variety of scientific, technological, economic and political challenges to the development and implementation of new therapies for this important fungal infection. The goal of this commentary is to discuss these challenges as well as recent developments and approaches to surmounting them.

People with decreased T-cell immunity are at highest risk for the development of cryptococcosis. Accordingly, cryptococcosis is a major problem for people living with HIV/AIDS, the most common cause of reduced T-cell based immunity [2]. In many instances, people present to medical care with symptoms of cryptococcal meningoencephalitis and, as a result, are diagnosed with HIV/AIDS. Because untreated cryptococcal meningoencephalitis is uniformly fatal, these patients must first survive their cryptococcal infection if they are to take advantage of the truly remarkable advances in HIV treatment [3]. Indeed, recent clinical studies indicate that it is important to treat the cryptococcosis before initiating antiretroviral therapy because concurrent therapy leads to poorer outcomes [4]. One of the major global challenges to the treatment of cryptococcosis is the fact that the gold standard therapy is not available in many of the resource-limited regions with the highest burden of disease [5]. Amphotericin B must be administered intravenously and has a number of associated toxicities that require laboratory monitoring; put simply, amphotericin B requires medical infrastructure that is not feasible in some regions. Despite these issues, amphotericin $\mathrm{B}$ is not the major obstacle to providing the best treatment in resource-limited regions. 5-flucytosine, which has recently been shown in a landmark clinical study to improve outcomes relative to amphotericin B alone [6], is essentially not available in resource-limited regions [7].

Fluconazole is used as an alternative agent in many regions where amphotericin $\mathrm{B}$ and 5-flucytosine are not available. Fluconazole is much cheaper, readily available, orally administered and very safe, even at high doses. Unfortunately, clinical trials have shown that it is consistently inferior to amphotericin B-based regimens, even at very high doses [8]. This lack of efficacy appears to be related to its fungistatic rather than fungicidal mode of action. The combination of fluconazole with amphotericin B was not as effective as that with 5-flucytosine [6]. The newer azoles (voriconazole, posaconazole, isavuconazole) all have in vitro activity

\author{
Damian J Krysan \\ Departments of Microbiology/ \\ Immunology, University of Rochester \\ School of Medicine \& Dentistry, \\ Rochester, NY 14642, USA \\ and \\ Pediatrics, University of Rochester School \\ of Medicine \& Dentistry, Rochester, \\ NY 14642, USA \\ Tel.: +1 5852759729 \\ Fax: +1 5852731104 \\ damian_krysan@urmc.rochester.edu
}


against $C$. neoformans and small clinical trials and/or case series of patients treated with these agents have been reported [9]. At this point, it is not clear that these agents offer clear-cut advantages over fluconazole and more studies are needed to define their roles in the management of cryptococcosis.

\section{"The identification of new antifungal agents that have acceptable levels of human toxicity is challenging because both the host and pathogen are eukaryotes."}

From the above discussion, it should be clear that new approaches to the treatment of cryptococcal disease represent an important unmet clinical need. The treatment outcomes for amphotericin B and 5-flucytosine are roughly comparable to those of other invasive fungal infections and, thus, expanding access to those therapies would represent a significant advance. This combination still has significant toxicity issues and, even in resource-rich regions with extensive medical infrastructure, many patients do not complete this regimen. Ideally, a new agent could be developed and brought in to practice. To address the problems associated with the current therapies, such a drug must have the following properties: fungicidal activity; ability to penetrate the blood-brain barrier; amenable to oral administration; and low require little or no laboratory monitoring of toxicity. These are fairly stringent criteria, particularly when one considers the sobering thought that our species has only brought three classes of antifungal drugs into practice.

The identification of new antifungal agents that have acceptable levels of human toxicity is challenging because both the host and pathogen are eukaryotes. For example, the reason Saccharomyces cerevisiae, baker's yeast, has been such an important model organism is that the fundamental genetic, biochemical and cell biological processes of eukaryotes are highly conserved from yeast to humans. While this fact provides a wonderful system to study fundamental biology in a way relevant to humans, it also means that what is bad for a fungus is typically bad for a human. In some ways, the search for new antifungal drugs has many parallels with cancer therapeutic development: the goal is to kill a eukaryotic pathogen lurking within a eukaryotic host.

To further complicate matters, the goal of most efforts has been to identify molecules with activity against the entire range of human fungal pathogens. In some ways, this approach is similar to requiring a new anticancer agent to be active against all human tumors. This point is particularly relevant to Cryptococcus since it is a Basidiomycete that has diverged significantly from Ascomycetes such as Candida and Aspergillus spp. Most antifungal drug discovery has focused on Ascomycetes rather than Basidiomycetes. Recently, we and others have compared the chemical and chemicalgenetic profiles of Cryptococcus to S. cerevisiae and C. albicans and found significant differences $[10,11]$. A clinically relevant example of these differences is the fact that the echinocandins, the most recently developed class of antifungal drugs, inhibit $C$. neoformans glucan synthase quite potently but are not clinically useful for therapy [12]. Such distinctions in the activity of antibiotics against species and classes of bacteria are well recognized; as a result, screens for agents active against Gram-positive or Gram-negative organisms are frequently performed. These considerations would suggest that directly screening $C$. neoformans is likely the most sensitive strategy for identifying new anticryptococcal agents. Although cryptococcal specific screens have not been widely reported in the past, the number of primary drug screens using $C$. neoformans has increased recently $[10,13,14]$. Hopefully, these efforts will lead to the development of new agents with activity against Cryptococcus.

The time-frame for the progression of a new antiinfective from preclinical discovery through clinical evaluation to use in clinical practice has historically been quite long. One approach to accelerating this process is to repurpose existing medications to new indications based on their so-called 'off-target' biological activities. As reviewed elsewhere [15], there have been a number of successful examples of this approach in a variety of areas of medicine. We and others have adopted this approach to identifying molecules with anticryptococcal activity and the pharmacological properties necessary to be useful in the treatment of cryptococcal disease: CNS penetration and oral bioavailability $[10,16]$. From these studies, antidepressants, antipsychotics and estrogen receptor antagonists have emerged as particularly promising candidates for repurposing as potential leads for anticryptococcal agents. Of these, sertraline, an antidepressant, has been evaluated in an open-label clinical trial in HIV-infected individuals with cryptococcal meningitis comparing standard therapy (amphotericin B followed by oral fluconazole) plus a range of sertraline doses to historical controls [17]. The sertraline-treated patients showed an increased rate of clearance of Cryptococcus from cerebrospinal fluid than observed in historical controls. This promising observation has led to the initiation of a Phase III study to determine the value of sertraline as an adjuvant to current therapy. Other repurposed drugs have similarly promising profiles. It is hoped that additional trials will be undertaken to test their clinical utility since this approach seems to be the 
most expedient route to much-needed improvements in anticryptococcal therapy.

In summary, although there are many challenges inherent in the quest to improve current methods in cryptococcal therapy, future prospects for solving this problem seem more promising than the situation only a few years ago because more and more investigators have entered the arena and are pursuing solutions in very creative ways.

\section{References}

1 Brown GD, Denning DW, Gow NA, Levitz SM, Netea MG, White TC. Hidden killers: human fungal infections. Sci. Transl. Med. 19(4), 165 (2012).

2 Park BJ, Wannemuehler KA, Marston BJ, Govender N, Pappas PG, Chiller TM. Estimation of the current global burden of cryptococcal meningitis among persons living with HIV/AIDS. AIDS 23(4), 525-530 (2009).

3 Sloan DJ, Dedicoat MJ, Lalloo DG. Treatment of cryptococcal meningitis in resource-limited settings. Curr. Opin. Infect. Dis. 22(5), 455-463 (2009).

4 Boulware DR, Meya DB, Muzoora C et al. Timing of antiretroviral therapy after diagnosis of cryptococcal meningitis. N. Engl. J. Med. 370(26), 2487-2498 (2014).

5 Loyse A, Thangaraj H, Easterbrook P et al. Cryptococcal meningitis: improving access to essential antifungal medicines in resource-poor countries. Lancet Infect. Dis. 13(7), 629-637 (2013).

6 Day JN, Chau TT, Wolbers M et al. Combination antifungal therapy for cryptococcal meningitis. N. Engl. J. Med. 368(14), 1291-1302 (2013).

7 Loyse A, Dromer F, Day J, Lortholary O, Harrison TS. Flucytosine and cryptococcosis: time to urgently address the worldwide accessibility of a 50-year-old antifungal. J. Antimicrob. Chemother. 68(11), 2435-2444 (2013).

8 Jarvis JN, Bicanic T, Loyse A et al. Determinants of mortality in a combined cohort of 501 patients with HIV-associated Cryptococcal meningitis: implications for improving outcomes. Clin. Infect. Dis. 58(5), 736-745 (2014).

9 Thompson GR 3rd, Rendon A, Dos Santos RR et al. Isavuconazole treatment of cryptococcosis and dimorphic mycoses. Clin. Infect. Dis. pii: ciw305 (2016) (Epub ahead of print).

\section{Financial \& competing interests disclosure}

The author has no relevant affiliations or financial involvement with any organization or entity with a financial interest in or financial conflict with the subject matter or materials discussed in the manuscript. This includes employment, consultancies, honoraria, stock ownership or options, expert testimony, grants or patents received or pending, or royalties.

No writing assistance was utilized in the production of this manuscript.

10 Butts A, DiDone L, Koselny K et al. A repurposing approach identifies off-patent drugs with fungicidal cryptococcal activity, a common structural chemotype, and pharmacological properties relevant to the treatment of cryptococcosis. Eukaryot. Cell 12(2), 278-287 (2013).

11 Brown JC, Nelson J, VanderSluis B et al. Unraveling the biology of a fungal meningitis pathogen using chemical genetics. Cell 159(5), 1168-1187 (2014).

12 Maligie MA, Selitrennikoff CP. Cryptococcus neoformans resistance to echinocandins: (1,3) beta-glucan synthase activity is sensitive to echinocandins. Antimicrob. Agents Chemother. 49(7), 2851-2856 (2005).

13 Rabjohns JL, Park YD, Dehdashti J et al. A high-throughput screening assay for fungicidal compounds against Cryptococcus neoformans. J. Biomol. Screen. 19(2), 270-277 (2014).

14 Hartland K, Pu J, Palmer M et al. High-throughput screen in Cryptococcus neoformans identifies a novel molecular scaffold that inhibits cell wall integrity pathway signaling. ACS Infect. Dis. 2(1), 93-102 (2016).

15 Frail DE, Brady M, Escott KJ et al. Pioneering governmentsponsored drug repositioning collaborations: progress and learning. Nat. Rev. Drug Discov. 14(12), 833-841 (2015).

16 Zhai B, Wu C, Wang L, Sachs MS, Lin X. The antidepressant sertraline provides a promising therapeutic option for neurotropic cryptococcal infections. Antimicrob. Agents Chemother. 56(7), 3758-3766 (2012).

17 Rhein J, Morawski BM, Hullsiek KH et al. Efficacy of adjunctive sertraline for the treatment of HIV-associated cryptococcal meningitis: an open-label dose-ranging study. Lancet Infect. Dis. 16(7), 809-818 (2016) (Epub ahead of print). 